\title{
Large Scale Structure and Supersymmetric Inflation Without Fine Tuning
}

\author{
G. Dvali \\ Dipartimento di Fisica \\ Universita di Pisa and INFN \\ Sezione di Pisa, I-56100 \\ Pisa, Italy \\ Q. Shafi and R. Schaefer \\ Bartol Research Institute, University of Delaware \\ Newark, DE 19716, USA
}

\begin{abstract}
We explore constraints on the spectral index $n$ of density fluctuations and the neutrino energy density fraction $\Omega_{H D M}$, employing data from a variety of large scale observations. The best fits occur for $n \approx 1$ and $\Omega_{H D M} \approx 0.15-0.30$, over a range of Hubble constants $40-60$ $\mathrm{km} \mathrm{s}^{-1} \mathrm{Mpc}^{-1}$. We present a new class of inflationary models based on realistic supersymmetric grand unified theories which do not have the usual 'fine tuning' problems. The amplitude of primordial density fluctuations, in particular, is found to be proportional to $\left(M_{X} / M_{P}\right)^{2}$, where $M_{X}\left(M_{P}\right)$ denote the GUT (Planck) scale, which is reminiscent of cosmic strings! The spectral index $n=0.98$, in excellent agreement with the observations provided the dark matter is a mixture of 'cold' and 'hot' components.
\end{abstract}

PACS Nos: 98.80Cq, 98.65Dx, 12.10Dm, 11.30Pb 
Recent studies of large scale structure formation [1], when confronted with a variety of data from the Cosmic Background Explorer [2] (COBE) and other large scale galaxy surveys, provide support for an inflationary scenario [3] in which the spectral index of density fluctuations $n$ is close to unity and the dark matter is a mixture of cold and hot components. Non-supersymmetric grand unified theories which give rise to precisely this scenario were constructed more than a decade ago [4. However, several fundamental challenges, including unification of GUTS with gravity and the gauge hierarchy problem, strongly hint that the supersymmetric grand unified (SUSY GUTS) framework may be a more promising way to proceed.

Supersymmetric GUTS based on $G \equiv S U(3)_{c} \times S U(3)_{L} \times S U(3)_{R}$ have the desirable feature that they permit unification of the standard model gauge couplings to occur at scales on the order of $10^{16} \mathrm{GeV}$, which is indicated by the recent LEP data. Moreover, as has recently been shown [5], by introducing either some symmetries, or alternatively R-symmetry, the gauge hierarchy ('fine tuning') problem can be resolved in SUSY GUTS based on G. It should be stressed that this is accomplished without complicating the 'higgs sector' of the theory. The 'minimal' choice will do, which is well nigh impossible in GUTS such as $S U(5)$ or $S O(10)$. The additional symmetries also ensure that the proton is essentially stable.

Encouraged by these developments, we investigate here if the inflationary scenario can be realized within the framework of $G$. We would call the attempt 'successful' if the following conditions are met. First, the scalar (higgs) sector of the theory, including the inflaton part, is determined by particle physics considerations. Second, no 'fine tuning' of parameters is needed. [This includes the gauge hierarchy problem.] Finally, the scenario must be such that the Planck scale corrections from supergravity and/or superstrings can be safely ignored. Although non-trivial, it turns out that all of these constraints can be satisfied within the framework of $G$. The inflationary scenario we are led to has previously been considered in general terms by Linde [6] (see also ref. [7]), and been dubbed 'hybrid' inflation. Our realization of 'hybrid inflation' within a supersymmetric framework is unique in a number of ways and can be implemented in a variety of models, especially those based on $G$. One particularly important result has to do with the amplitude of primordial density fluctuations, which turns out to be proportional to $\left(M_{X} / M_{P}\right)^{2}$, where $M_{X}$ denotes a superheavy (GUT scale) and $M_{P} \simeq 1.2 \times 10^{19} \mathrm{GeV}$ is the Planck mass. The spectral index of the 
density fluctuations is very close to unity as required by the observations.

We begin with a definition of the cosmological parameters in the simplest inflationary models.

1. The density $\rho$ is the critical density $\rho_{c}=3 H_{0}^{2} /(8 \pi G)$, where $H_{0}=100 h$ $\mathrm{km} \mathrm{s}^{-1} \mathrm{Mpc}^{-1}$ is the present value of the Hubble constant and $G$ is Newton's gravitational constant.

2. We take $h=0.5 \pm 0.1$, which corresponds to the range of $h$ allowed by the combination of observations and constraints on the age of the universe in critical density models with a vanishing cosmological constant.

3. We take the baryon fraction to be the midpoint of the range allowed by big bang nucleosynthesis [8], $\Omega_{\text {baryon }} h^{2}=0.0125 \pm 0.0025$, where $\Omega_{\text {baryon }} \equiv \rho_{\text {baryon }} / \rho_{c}$.

4. The remaining mass density is in the dark matter which is composed of some mixture of cold (CDM) and hot (HDM) components. The latter is assumed to be "lightly" massive (few eV) relic neutrinos. The relative concentrations of the two components is left to be determined by the data. We include the possibility that the dark matter may be all CDM.

5. The power spectrum, which is the Fourier transform of the two-point density autocorrelation function, has the primordial form $P(k) \propto k^{n}$, where $k$ is the amplitude of the Fourier wavevector, and $n$ denotes the spectral index. The value $n=1$ corresponds to the Harrison-Zeldovich spectrum.

The above set of parameters define a family of inflationary models. Their ultimate test comes from comparison to data. Here we will extend the treatment previously described in Ref. [1] to include larger values of the density fluctuation spectral index $n$ and different values of the Hubble constant. We will give a brief summary of the procedure used there and present the new results in Figure 1. The data we use is summarized next.

We begin with the data from COBE. The amplitude is most conveniently characterized in a relatively $n$ independent way by the expected hexadecupole moment of the temperature anisotropy $\delta T_{4}=12.8 \pm 2.3 \mu \mathrm{K}$, where the error includes effects such as radiometer noise and cosmic variance [2]. 
(This treatment is consistent with the recent analysis of the COBE two year data [9].) This data set probes the spectrum on the largest scales $\left(\sim 10^{3} h^{-1}\right.$ $\mathrm{Mpc}$ ). For smaller scales, we use the data obtained from large galaxy surveys, particularly that of the Infrared Astronomy Satellite (IRAS) catalogue of galaxies. In ref. 10 this catalogue was used to obtain a direct estimate of the power spectrum in the range $30 h / \mathrm{Mpc}<2 \pi / k<300 h / \mathrm{Mpc}$. The resulting power spectrum is in good agreement with estimates from other surveys based on optically selected galaxies. The IRAS catalogue of galaxies has also been used in a different way to determine the large scale bulk velocity field. We use the values given by the potential flow algorithm ("POTENT") collaboration (see ref. [11]).

There are important constraints on the power spectrum which come from structures which have evolved so that their description is inherently nonlinear. If we make some basic assumptions about the formation of these structures, we can use this data to constrain the amplitudes of the fluctuations in the linear theory. We will use two such pieces of information. First, in pre-COBE days the normalization of power spectra was usually done by specifying the mass fluctuation on $8 h^{-1}$ Mpc scales, and much effort has been made to determine this normalization from the data. In ref [12, an attempt was made to estimate this quantity from the abundance of clusters. Estimates of the mass fluctuation on $8 h^{-1} \mathrm{Mpc}$ from different galaxy surveys yield results that are consistent [13]. We adopt the synthesized value $\delta M / M\left(8 h^{-1} \mathrm{Mpc}\right)<0.8$ as a constraint on the amplitude of linear perturbations.

Second, the number of early quasars at high redshift requires a minimum amplitude of mass fluctuation. This issue has recently been extensively studied [14]. In Ref. [1] two of the present authors estimated the error on this amplitude introduced by theoretical modeling uncertainties and found that the lower limit on the amplitude of mass fluctuations required to make the early quasar population is $\delta M / M\left(0.6 h^{-1} \mathrm{Mpc}\right) \geq 1.1 \pm 0.2$. We will use this constraint here as well [15].

The data are then analyzed in the following way. For a given value of $n$ and a hot dark matter fraction $\Omega_{H D M}$, we calculate up to a normalization factor, the hexadecupole moment of relic temperature fluctuations, the power spectrum for the IRAS power spectrum wavenumbers, the large scale velocities, the linear mass fluctuation on $8 h^{-1} \mathrm{Mpc}$, and the amplitude of the quasar sized mass fluctuations. In addition, while most of our list of 
constraints depend on the mass fluctuations, the variations in galactic number can be somewhat different than mass fluctuations. To compare our mass fluctuation power spectrum with galactic number we have to also introduce a "density bias factor" $b_{I}$ to allow for the possibility the IRAS galaxies may not trace the mass. The fact that the depth coordinate in the galaxy survey is doppler redshift space rather than physical length introduces some effects which must be corrected for (see ref. [16]). For each value of $\Omega_{H D M}$ and $n$ we first do a least squares fit of the normalization and the bias factor $b_{I}$.

After fitting these parameters, we proceed to calculate the value of the $\chi^{2}$ (goodness of fit) statistic from the data for each model. For the bulk velocities, we have combined the uncertainty due to "cosmic variance" in quadrature with the quoted analysis errors, because the velocities correspond to a very limited sampling of a Gaussian velocity field. After finding the best fitting model, we record how the $\chi^{2}$ statistic changes as we vary the parameters $n$ and $\Omega_{H D M}$ and draw confidence level contours in the $n-\Omega_{H D M}$ plane [17]. We repeat this entire procedure for different values of the Hubble constant.

Figure one dramatically shows how the data favor values of $n$ very close to unity with the actual limits depending somewhat on the mix of dark matter and the Hubble constant. Overall we can say that with $99 \%$ confidence, $0.80<n\left[H_{0} /\left(50 \mathrm{~km} \mathrm{~s}^{-1} \mathrm{Mpc}^{-1}\right)\right]^{1 / 2}<1.15$, independent of the dark matter composition. The value of $n=1$ works well over the range of Hubble constants $40-60 \mathrm{~km} \mathrm{~s}^{-1} \mathrm{Mpc}^{-1}$. It appears that $n$ is constrained to be quite close to unity by the data.

We now discuss how such density fluctuations can be realized in realistic supersymmetric GUTS. We are particularly interested in identifying models in which there are no 'fine tuning' (including gauge hierarchy) problems. To set things up, consider the following globally supersymmetric renormalizable superpotential $W$ :

$$
W=\kappa S \bar{\phi} \phi-\mu^{2} S
$$

where $\phi(\bar{\phi})$ denote a conjugate pair of superfields transforming as non-trivial representations of some gauge group, while $S$ is a gauge singlet superfield. This superpotential is 'natural' in the strong sense [18]. It is of the most general form consistent with R-symmetry under which $S \rightarrow e^{i \gamma} S, W \rightarrow$ $e^{i \gamma} W$, while the product $\bar{\phi} \phi$ is invariant. Note that cubic terms in $\phi$ and $\bar{\phi}$ can be forbidden by assuming, for example, the transformations $\phi \rightarrow \bar{e}^{i \gamma} \phi, \bar{\phi} \rightarrow$ 
$e^{i \gamma} \bar{\phi}$. In realistic models such terms may be allowed without altering the main conclusions.

We point out that, at least in the global SUSY case, the R-symmetry is the unique choice for implementing the 'false' vacuum inflationary scenario in a natural way. It is the only symmetry which can eliminate all of the undesirable self-couplings of the (inflaton) $S$, while allowing the linear term in the superpotential. With supersymmetry unbroken, the potential takes the form (we represent the scalar components with the same symbols as the superfields if there is no danger of confusion!):

$$
V(S, \phi, \bar{\phi})=\kappa^{2}|S|^{2}\left[|\phi|^{2}+|\bar{\phi}|^{2}\right]+\left|\kappa \phi \bar{\phi}-\mu^{2}\right|^{2}+D-\text { terms }
$$

The $D$-terms vanish along the (D-flat) direction $|\phi|=\left|\bar{\phi}^{*}\right|$. Consequently, the only supersymmetric minimum of the potential is at

$$
\begin{gathered}
<S>=0 \\
M_{X} \equiv\langle|\phi|\rangle=\langle|\bar{\phi}|\rangle=\mu / \sqrt{\kappa}(\mu>0, \kappa>0)
\end{gathered}
$$

We will say more about the scale $M_{X}$ shortly.

Consider now an early universe scenario with chaotic initial conditions. For $|S|>\left|S_{c}\right|=\mu / \sqrt{\kappa}$, the effective potential $V$ is minimized by $\langle\phi\rangle=\langle\bar{\phi}\rangle=0$. That is, for $|S|>S_{c}$, the energy density is dominated by the 'false' vacuum energy density $\mu^{4}$, which can therefore lead to an exponentially expanding (inflationary) universe. The potential in (2) does not contain a term which can drive $S$ to its minimum value. This, however, is no longer the case when the quantum corrections are taken into consideration.

With $|S|>S_{c}$, both $\phi$ and $\bar{\phi}$ vanish and there is a nonzero $F_{S}-\operatorname{term}(=$ $\mu^{2}$ ) which breaks supersymmetry, such that the one loop corrections to the effective potential are non-vanishing, and given by [19]

$$
\Delta V(S)=\sum_{i} \frac{(-1)^{F}}{64 \pi^{2}} M_{i}(S)^{4} \ln \left(\frac{M_{i}(S)^{2}}{\Lambda^{2}}\right)
$$

where the summation is over all helicity states, $(-1)^{F}$ indicates that the bosons and fermions make opposite sign contributions, and $\Lambda$ denotes a renormalization mass. The quantum corrections will help drive $S$ to its minimum.

Note that for $S>S_{c}$ there is no mass splitting inside the gauge supermultiplets or the $S$-superfield (actually the masses of $S$-scalar and its fermionic 
superpartner both vanish). The non-vanishing contribution is from the mass splitting within the $\phi, \bar{\phi}$ superfields. The complex scalars in $\phi, \bar{\phi}$ are split by the nonzero $F_{S}$-term into two pairs of real scalar and pseudoscalar components with mass squared $\kappa^{2} S^{2} \pm \kappa \mu^{2}$, whereas the fermionic partners have mass $\kappa S$. The one loop corrected effective potential (along the inflationary trajectory $S>S_{c}, \phi=\bar{\phi}=0$ ) is given by

$$
\begin{array}{r}
V_{e f f}(S)=\mu^{4}+\frac{\kappa^{2}}{32 \pi^{2}}\left[2 \mu^{4} \ln \left(\frac{\kappa^{2}|S|^{2}}{\Lambda^{2}}\right)+\right. \\
\left.\left(\kappa S^{2}-\mu^{2}\right)^{2} \ln \left(1-\frac{\mu^{2}}{\kappa S^{2}}\right)+\left(\kappa S^{2}+\mu^{2}\right)^{2} \ln \left(1+\frac{\mu^{2}}{\kappa S^{2}}\right)\right]
\end{array}
$$

If $S$ is sufficiently greater than $S_{c}, V_{\text {eff }}(S)$ reduces to the simpler form

$$
V_{e f f}\left(S \gg S_{c}\right) \approx \mu^{4}\left[1+\frac{\kappa^{2}}{32 \pi^{2}}\left(\ln \frac{\kappa^{2} S^{2}}{\Lambda^{2}}+\frac{3}{2}\right)\right]
$$

For $|S|>S_{c}$, the inflationary phase is dominated by the false vacuum energy $\mu^{4}$ as in the tree level case, but the additional contribution in (5) will now drive $S$ to its minimum. The GUT phase transition takes place only after the $S$ field drops to its critical value $S_{c}\left(=M_{X}\right)$. Below $S_{c}$, the $S$ field is driven to zero by the positive mass term $\kappa^{2}|S|^{2}\left|\phi^{2}\right|$ which is increasingly more effective due to the increase of the $\phi, \bar{\phi}$ vevs (induced by the decreasing $S$ ). All of the fields rapidly adjust to their vacuum values (3), thereby restoring supersymmetry.

Note that the end of inflation does not necessarily coincide with the GUT phase transition which occurs when $S$ approaches $S_{c}$. The end is signaled when the "slow roll" condition is violated for some $S>S_{c}$. We can characterize the "slow roll" condition as (see first paper in ref. [0])

$$
\epsilon<<1,|\eta|<<1
$$

where

$$
\epsilon=\frac{M_{P}^{2}}{16 \pi}\left(\frac{V^{\prime}}{V}\right)^{2}, \eta=\frac{M_{P}^{2}}{8 \pi} \frac{V^{\prime \prime}}{V}
$$

(the prime refers to derivatives with respect to $S$ ). The inflationary phase may end before the GUT transition if the above conditions are violated at some $S>S_{c}$. For convenience, we can use the parametrization $S=x S_{c}$, 
where the parameter $x$ characterizes the rolling of $S$. (The GUT phase transition occurs for $x=1$.) The quantities $\epsilon$ and $\eta$ are given by

$$
\begin{gathered}
\epsilon=\left(\frac{\kappa^{2} M_{P}}{16 \pi^{2} M_{X}}\right)^{2} \frac{x^{2}}{16 \pi}\left[\left(x^{2}-1\right) \ln \left(1-\frac{1}{x^{2}}\right)+\left(x^{2}+1\right) \ln \left(1+\frac{1}{x^{2}}\right)\right]^{2} \\
\eta=\left(\frac{\kappa M_{P}}{4 \pi M_{X}}\right)^{2} \frac{1}{8 \pi}\left[\left(3 x^{2}-1\right) \ln \left(1-\frac{1}{x^{2}}\right)+\left(3 x^{2}+1\right) \ln \left(1+\frac{1}{x^{2}}\right)\right]
\end{gathered}
$$

Note that $\eta$ becomes infinitely large for $x=1$, so that inflation ends as $x$ approaches 1 (from above).

So far, we have not introduced any supersymmetry violation in the system (the global minimum in (3) is supersymmetric). In conventional schemes (say $\mathrm{N}=1$ supergravity), this breaking is introduced through the soft SUSY violating terms in the tree level potential. The main influence of such terms on the inflationary scenario discussed above arises from the fact that the SUSY breaking induces a TeV scale (mass) ${ }^{2}$-term for the scalars, in particular for the $S$ field. The term $m^{2}|S|^{2}(m \sim$ TeV) provides an extra force driving $S$ to the minimum. However, unless the coupling constant $\kappa$ is very small, the soft mass terms only provide a small correction to $V_{\text {eff }}(S)$ in (5), and so cannot significantly affect the above dynamics. This is not surprising since for $|S|>S_{c}$, the non-supersymmetric (mass) ${ }^{2}$ splitting inside the $\phi, \bar{\phi}$ superfields is $\kappa \mu^{2}$ which, as we shall see, is much larger then $m^{2}$. In such a situation the inflationary scenario above is practically independent of the particular mechanism of supersymmetry breaking.

Let us now compare the predicted quadrupole anisotropy, based on (5), with the value $\left(\approx 7 \times 10^{-6}\right)$ measured by COBE. From the scalar density fluctuations one has (see first paper in ref. [0])

$$
\begin{aligned}
\left(\frac{\Delta T}{T}\right)_{Q} & \left.\approx \sqrt{\frac{32 \pi}{45}} \frac{V^{\frac{3}{2}}}{V^{\prime} M_{P}^{3}}\right|_{x_{Q}} \\
& \approx\left(8 \pi N_{Q}\right)^{\frac{1}{2}}\left(M_{X} / M_{P}\right)^{2}
\end{aligned}
$$

where the subscript $x_{Q}$ indicates the value of $S$ as the scale (which evolved to the present horizon size) crossed outside the de Sitter horizon during inflation, and $N_{Q}(\approx 50-60)$ denotes the appropriate number of e-foldings. The formula in (10) is remarkable in that the fluctuation amplitude is proportional to $\left(M_{X} / M_{P}\right)^{2}$, just as in the cosmic string scenario! The amplitude turns out to be in the right ball park, without having to 'fine tune' additional 
parameters (such as dimensionless quartic couplings and/or the mass of the inflaton). Using (10), we can estimate the fundamental parameter $M_{X}$ to be on the order of $10^{15.5} \mathrm{GeV}$. We have ignored the contribution of the tensor fluctuations to the anisotropy, because they are suppressed by a factor of $\kappa /\left[8 \pi\left(N_{Q}\right)^{1 / 2}\right]$ relative to the scalar component in eq. (10).

The spectral index $n$ of the density fluctuations is given by

$$
n \simeq 1-\frac{1}{N_{Q}} \simeq 0.98
$$

which, as we have seen earlier, is in the central range of the values preferred by observations.

An estimate of the coupling $\kappa$ is obtained from the relation

$$
\frac{\kappa}{x_{Q}} \sim \frac{8 \pi^{3 / 2}}{\sqrt{N_{Q}}} \frac{M_{X}}{M_{P}}
$$

With $x_{Q} \sim 10$ say, (which corresponds to $S \sim 10^{16.5} \mathrm{GeV}$, and the Planck scale corrections can be safely ignored), the coupling $\kappa$ turns out to be on the order of $10^{-2}$. Note that for this value of $\kappa$, the tensor generated anisotropies are less than $10^{-4}$ of the scalar anisotropy amplitude.

Having outlined how supersymmetric models can lead to a successful inflationary scenario without invoking small dimensionless couplings, we now proceed to discuss how this idea can be realized in realistic SUSY GUTS. We would like the model to be well motivated from the particle physics viewpoint. For instance, a non-trivial constraint would be the absence of the gauge hierarchy and proton decay problems. Although this constraint presumably can be met in GUTS such as $S U(5)$ or $S O(10)$, the higgs sector of the theory becomes quite messy. An even more formidable constraint stems from the fact that the phase transition involving the gauge non-singlet fields $(\phi, \bar{\phi})$ occurs at the end of inflation. In $S U(5)$ this would lead to the monopole problem. We therefore must resort to SUSY GUTS with rank 5 or higher in order that at least the possibility exists for the monopoles to be inflated away.

The simplest example of SUSY GUTS we are aware of in which the gauge hierarchy problem can be resolved with a minimal higgs system is based on $G\left(\equiv S U(3)_{c} \times S U(3)_{L} \times S U(3)_{R}\right)$. Under the gauge group $G$, the left 
handed lepton, quark and antiquark superfields respectively transform as $(1, \overline{3}, 3),(3,3,1)$ and $(\overline{3}, 1, \overline{3})$. They are denoted as $\lambda_{i}, Q_{i}$ and $Q_{i}^{c}(i=1,2,3)$ :

$$
\begin{gathered}
\lambda_{i}=\left(\begin{array}{ccc}
H^{(1)} & H^{(2)} & L \\
e^{c} & \nu^{c} & N
\end{array}\right) \\
Q_{i}=\left(\begin{array}{c}
u \\
d \\
g
\end{array}\right) \\
Q_{i}^{c}=\left(\begin{array}{lll}
u^{c} & d^{c} g^{c}
\end{array}\right)
\end{gathered}
$$

Here $H^{(1)}, H^{(2)}$ and $L$ denote $S U(2)_{L}$ doublet superfields, $e^{c}$ is an $S U(2)_{L}$ singlet, $N$ and $\nu^{c}$ are standard model singlets, while $g\left(g^{c}\right)$ denote additional down-type quark (antiquark) superfields. The $S U(3)_{L}\left(S U(3)_{R}\right)$ symmetry acts along columns (rows). The symmetry breaking of $G$ to $S U(3)_{c} \times U(1)_{\mathrm{em}}$ requires at least two sets of higgs supermultiplets, $\lambda+\bar{\lambda}$ and $\lambda^{\prime}+\bar{\lambda}^{\prime}$, where $\lambda\left(\lambda^{\prime}\right)$ transform as $\lambda_{i}$ above. The conjugate fields $\bar{\lambda}\left(\bar{\lambda}^{\prime}\right)$ are needed to ensure that the SUSY breaking scale is well below the GUT scale and that the anomalies cancel.

Imposition of a suitable R-symmetry, largely determined by the requirement that a pair of electroweak doublets (in $\lambda$ ) remains light, fixes the renormalizable part of the superpotential as follows (we will not give the couplings involving the chiral fermion families):

$$
\begin{aligned}
W & =W_{\lambda}+W_{\lambda^{\prime}}+W_{\lambda \lambda^{\prime}} \\
W_{\lambda} & =\kappa S \lambda \bar{\lambda}-\mu^{2} S+a \bar{\lambda}^{3} \\
W_{\lambda^{\prime}} & =b \lambda^{\prime 3}+c \bar{\lambda}^{\prime 3} \\
W_{\lambda \lambda^{\prime}} & =d \bar{\lambda}^{2} \bar{\lambda}^{\prime}+e \bar{\lambda} \bar{\lambda}^{\prime 2}
\end{aligned}
$$

Here the bilinear and trilinear terms stand for the appropriate $G$ invariant combinations. For instance, $\lambda \bar{\lambda} \equiv \operatorname{Tr}\left(\lambda_{\alpha}^{A} \bar{\lambda}_{A}^{\alpha}\right), \lambda^{\prime 3} \equiv \epsilon^{\alpha \beta \gamma} \epsilon_{A B C} \lambda_{\alpha}^{\prime A} \lambda_{\beta}^{\prime B} \lambda_{\gamma}^{\prime C}$, etc., where the Greek (Latin) indices refer to $S U(3)_{L}\left(S U(3)_{R}\right)$. The R-charges of the superfields are as follows: $S(1), \lambda\left(-\frac{1}{3}\right), \bar{\lambda}\left(\frac{1}{3}\right), \lambda^{\prime}\left(\frac{1}{3}\right), \bar{\lambda}^{\prime}\left(\frac{1}{3}\right)$, where $W \rightarrow$ $e^{i \theta} W$ under $\mathrm{R}$. The superlarge vevs will be oriented along the directions

$$
|\lambda|=\left|\bar{\lambda}^{*}\right|=\left(\begin{array}{ccc}
0 & 0 & 0 \\
0 & 0 & 0 \\
0 & 0 & N
\end{array}\right)
$$




$$
\left|\lambda^{\prime}\right|=\left|\bar{\lambda}^{* *}\right|=\left(\begin{array}{ccc}
0 & 0 & 0 \\
0 & 0 & 0 \\
0 & \nu^{c \prime} & 0
\end{array}\right)
$$

The absence of a $\lambda^{3}$ term in (14) guarantees that the electroweak doublet pair $\left(H^{(1)}-H^{(2)}\right)$ in $\lambda$ remains massless at tree level, despite the GUT symmetry breaking. The well known ' $\mu$-term' for this pair is generated by the nonrenormalizable coupling $\lambda^{3}\left(\lambda^{\prime} \bar{\lambda}^{\prime}\right)^{3} / M^{6},\left(M \equiv M_{P} / \sqrt{8 \pi} \sim 10^{18} \mathrm{GeV}\right)$ which is the leading contribution allowed by the R-symmetry. With $\left\langle\lambda^{\prime}>/ M \sim\right.$ $10^{-2}, \mu$ is in the TeV range as desired. [Note that without the $W_{\lambda, \lambda^{\prime}}$ term, the superpotential in (14) would be invariant under $\left[S U(3)_{L} \times S U(3)_{R}\right]^{2}$. $]$

It is important to point out that in addition to resolving the gauge hierarchy problem, the R-symmetry guarantees the absence of dimension four and five baryon number violating operators. That is, cubic superpotential couplings such as $Q_{i} Q_{j} Q_{k}$ are absent, where the $Q_{i}$ 's and $Q_{i}^{c}$ 's carry R-charges $=\frac{2}{3}$. The proton is essentially stable according to this model. Moreover, the desired couplings such as $Q_{i} Q_{j}^{c} \lambda$, which provide masses for the quarks, are allowed. Similarly, there are no undesirable lepton number violating couplings.

The superpotential (14) is the generalization of (1), on which the inflationary scenario was based, to a realisitic SUSY GUT model. Without going into the details, which will be published elsewhere, we briefly summarize how things work out. The role of the $\phi-\bar{\phi}-S$ sector in (1) is played by the superfields $\lambda-\bar{\lambda}-S$. [Note that the $\bar{\lambda}^{3}$ term in (14) vanishes along the vacuum direction (15).] Prior to inflation, the vevs in the $\lambda^{\prime}-\bar{\lambda}^{\prime}$ sector, which arise from an interplay of suitable non-renormalizable couplings and supersymmetry breaking mass terms, break the $(S U(3))^{3}$ GUT symmetry to $S U(3)_{c} \times S U(2)_{L} \times S U(2)_{R}^{\prime} \times U(1)$. The subsequent inflationary phase driven by the $S$ field inflates away the monopoles produced during the first $\left(\lambda^{\prime}-\bar{\lambda}^{\prime}\right)$ breaking. The $\lambda-\bar{\lambda}$ fields acquire non-zero vevs $\left(\sim 10^{15.5} \mathrm{GeV}\right)$ at the end of inflation.

An inflationary scenario is incomplete without some discussion of i) how the baryon asymmetry is generated and ii) the dark matter candidate(s). As far as (i) is concerned, it has been shown in ref. [5] that the resolution of the gauge hierarchy problem within the framework of $G$ necessarily leads to an additional pair of electroweak doublets. (Actually, one effectively ends up with a $5+\overline{5}$ of $S U(5)$, of mass $\sim T e V$. Unification of the three gauge 
couplings at scales $\sim 10^{16} \mathrm{GeV}$ is thereby preserved.) This strongly suggests generating the baryon asymmetry at the electroweak scale. The reheat temperature in this scheme is high, $\sim 10^{14} \mathrm{GeV}$, which means that the gravitino mass must be large in order that the primordial gravitino problem is avoided. Precisely how this works out would depend on how local supersymmetry is broken.

As far as dark matter is concerned, the lightest supersymmetric particle (LSP) is completely stable and so presents itself as an ideal 'cold' dark matter candidate. Indeed, one can speculate here about the nature of the LSP. The point is that in the absence of the two lighter families, the well known parameter $\tan \beta\left(\equiv<H^{u}>/<H^{d}>\right.$ ) of the MSSM is equal to $m_{t} / m_{b}$ at the GUT scale $M_{X}$. (This follows from the superpotential coupling $Q Q^{c} \lambda$.) Coupled with the radiative electroweak breaking scenario, this leads to a cold dark matter candidate essentially composed of the bino with mass in the range of $200-350 \mathrm{GeV}$.

What about the neutrinos in this scheme? The lepton chiral supermultiplets $\lambda_{i}(i=1,2,3)$ transform according to the same $G$ representation as the higgs supermultiplets $\lambda, \lambda^{\prime}$. The R-symmetry allows the coupling $\lambda_{i} \lambda_{j} \lambda$ provided that the R-charge of $\lambda_{i}$ is $\frac{2}{3}$. This is needed to provide mass say to the $\tau$ lepton. But it also gives a Dirac mass $m_{\tau} \tan \beta$ to the $\left(\nu_{\tau}\right.$ and $\left.\nu_{\tau}^{c}\right)$ neutrinos in $\lambda_{i}$. Now, the R-symmetry prevents one from giving a large Majorana mass to the $\nu^{c}$ fields, which could make the neutrinos unacceptably heavy. We are therefore led to introduce three G-singlet superfields $F_{i}$ (one per chiral family) in order that the standard see-saw mechanism can be implemented. One of the neutrinos can now have a mass in the few electron volt as suggested by the data on large scale structure formation.

In conclusion, it is gratifying to learn that a satisfactory inflationary scenario, consistent with large scale structure observations, can be realized in realistic supersymmetric GUTS in which the sector that 'drives' inflation is determined by particle physics considerations, and no fine tuning of the parameters is needed.

Acknowledgement. We would like to gratefully acknowledge the support of this research by the DOE under grant DEFG02 - 91ER40626.

\section{References}


[1] R. Schaefer and Q. Shafi, Phys. Rev. D, 49, 4990, (1994).

[2] E.L. Wright, et al., Astrophys. J, 420, 1, (1994).

[3] A.D. Linde, Particle Physics and Inflationary Cosmology, Harwood Academic, Switzerland (1990); E.W. Kolb, and M.S. Turner, The Early Universe, Addison Wesley (1990).

[4] Q. Shafi and F.W. Stecker, Phys. Rev. Lett., 53, 1292, (1984).

[5] G. Dvali and Q. Shafi, Phys. Lett. B., 326, 258, (1994).

[6] A.D. Linde, Phys. Lett. B, 259, 38 (1991) and Stanford Preprint 93-17 (1993).

[7] A.R. Liddle and D.H. Lyth, Phys. Rep. 231, 1 (1993); E.J. Copeland et. al., Phys. Rev. D, in press; S. Mollenrach, S. Matarrese and F. Lucchin, CERN Preprint (1993).

[8] T. P. Walker, et al., Astrophys. J., 376, 51, (1991); M.S. Smith, L. H. Kawano, and R.A. Malaney, Astrophys. J. Suppl., 85, 219 (1993).

[9] K. Gorski et al., , COBE Preprint 94-08 (1994).

[10] Feldman, H., Kaiser, N., and Peacock, J.A., Mon. Not. R. Ast. Soc. (1993)

[11] A. Dekel, "Dynamics of Cosmic Flows", Ann. Revs. Astron. Astrop., 32, in press, (1994).

[12] S. D. M. White, G. Efstathiou, and C. S. Frenk, Mon. Not. Roy. Ast. Soc., 262, 1023, (1993).

[13] W. Saunders, M. Rowan-Robinson, and A. Lawrence, Mon. Not. Roy. Ast. Soc., 258, 134, (1992); K.B. Fisher, et al., Mon. Not. Roy. Ast. Soc., (in press).

[14] M. G. Haehnelt and M. J. Rees, Mon. Not. Roy. Ast. Soc., 263 168, (1993). R. K. Schaefer and Q. Shafi, Phys. Rev. D, 47, 1333, (1993); M. G. Haehnelt, Mon. Not. Roy. Ast. Soc., in press, (1994); A. Nusser and J. Silk, Astrophys. J. Lett., 411, L1, (1993). 
[15] It has been suggested that the number of "Lyman alpha clouds", objects which are detected by their Lyman series absorption lines in the continuum emission of quasars, may provide an even more stringent bound on the amplitude of galaxy sized density fluctuations. For a recent discussion and additional references see A. Klypin et al., preprint astro-ph/9405003, April 1994.

[16] N. Kaiser, 1987, Mon. Not. Roy. Ast. Soc., 227, 1.

[17] Y. Avni, Atrophys. J., 210, 642, (1976). See also W.H. Press, B. P. Flannery, S.A. Teukolsky, and W.T. Vetterling, Numerical Recipes, Cambridge University Press, Cambridge, (1986).

[18] E. Witten, Phys. Lett., 105B, 267 (1981).

[19] S. Coleman and E. Weinberg, Phys. Rev., D7, 1888 (1973).

\section{Figure Captions}

Figure 1. The $\chi^{2}$ contours (68 \%, 95\%, and 99\% confidence levels) for values of $n$ and $\Omega_{h d m}$ implied by the large scale structure data. In the left panel we have the constraints for a Hubble constant of $40 \mathrm{~km} \mathrm{~s}^{-1} \mathrm{Mpc}^{-1}$. Similarly we have the constraints for Hubble constants of 50 and $60 \mathrm{~km} \mathrm{~s}^{-1} \mathrm{Mpc}^{-1}$ in the center and right panels. Values of $n \sim 1$ are consistent with all values of the Hubble constant. We note that decreasing the Hubble constant favors larger values of $n$ and smaller $\Omega_{H D M}$. 
This figure "fig1-1.png" is available in "png" format from: http://arXiv.org/ps/hep-ph/9406319v1 